\title{
A Multi-level Hierarchical Game-Theoretical Approach for Cognitive Networks
}

\author{
Gaoning He \& Samson Lasaulce \\ Lab. des Signaux et Systemes \\ CNRS - Supélec - Paris Sud \\ 91192 Gif-sur-Yvette - FRANCE \\ gaoning.he@gmail.com \\ lasaulce@1ss.supelec.fr
}

\author{
Yezekael Hayel \\ Lab. Informatique d'Avignon \\ Universite d'Avignon \\ 84911 Avignon - France \\ yezekael.hayel@univavignon.fr
}

\author{
Mérouane Debbah \\ Alcatel-Lucent Chair \\ Supélec \\ 91192 Gif-sur-Yvette - France \\ merouane.debbah@supelec.fr
}

\begin{abstract}
This paper considers a game theoretical approach to study the problem of energy-efficient power control in cognitive wireless networks where some hierarchy exists between cognitive devices. This hierarchical structure is introduced either to model cognitive networks where some transmitters have more sensing capabilities than the others (they can observe more transmitters) or networks for which the degrees of knowledge about the network are different. The optimum selfish power control policy under consideration is determined in two scenarios: 1. $K$ transmitters with $K$ hierarchy levels; 2 . two hierarchy levels comprising $K_{1}$ non-cognitive terminals and $K-K_{1}$ cognitive terminals. For both scenarios, it is shown that the derived equilibria Pareto-dominate the one obtained without hierarchy. Interestingly, in scenario 1 we show that following is better than leading in terms of individual energy-efficiency. In scenario 2, considering the global network efficiency, we show that there exists an optimum number of cognitive terminals to put in the network.
\end{abstract}

\section{INTRODUCTION}

Over the past decade, more and more research works have been devoted to study decentralized or distributed wireless networks. There are many relevant concepts such as cognitive radio, unlicensed bands, ad hoc networks, scalable resource allocation and control policies, which play an important role in this development. In this paper, the problem of distributed power control is studied. The network is said to be distributed because each transmitter can choose freely his power control policy. As communications are mutually interfered at the receiver side and transmitters are free to decide their strategies, a natural analytical method is to model such a situation as a game problem [1]. In this game, transmitters are assumed to be selfish and maximize their energy-efficiency utilities. The first work analyzing this non-cooperative energy-efficient power control game is [2], in which a block fading multiple access channel is assumed and a static game model is used to model the power control problem on each block. This scenario has been extended to single-carrier systems with pricing in [3], to single-carrier systems under a Stackelberg formulation in [4], and to multi-carrier systems in [5].

The purpose of the present work is to provide several new results on the hierarchical formulation of the problem for single-carrier systems introduced in [4]. Note that all the results can be generalized, as a second step, to multi-carrier systems. The restriction to the first type of systems is made deliberately in order to present the new ideas and results as clearly as possible. There are many motivations for studying hierarchical wireless networks, but the most important ones in the framework of this paper are: improving the global network efficiency and modeling aspect. As explained in the abstract, hierarchy means that the knowledge levels of the transmitters are asymmetric. This would be a typical situation in wireless networks where only certain transmitters are cognitive and the others are not. More generally, this is the case in a network where a transmitter, say $k \in\{1, \ldots, K\}$ can sense or observe $N_{k}$ transmitters and the sensing capability numbers $N_{k}$ are different. In [4] the authors focus on a special case with one leader who cannot observe anyone (i.e., $N_{1}=0$ ) and $K-1$ followers who can only observe the leader (i.e., $\left.N_{k}=1, \forall k \leq 1\right)$. Here, we analyze two new scenarios which are more general and useful to the application of cognitive wireless networks. In the first scenario (see Section IV), there are $K$ hierarchy levels and $N_{1}=0, N_{2}=1, \cdots, N_{K}=K-1$ meaning that the leader (transmitter 1 ) is not equipped with cognitive radio, the follower at level 2 (transmitter 2) can observe the leader, ..., and finally the follower at level $K-1$ (transmitter $K-1$ ) is the one who has the greatest sensing capability and can sense everyone. In the second scenario, we assume that $N_{k}=0$ for $k \in\left\{1, \ldots, K_{1}\right\}$ and $N_{k}=1$ for $k \in\left\{K_{1}+1, \ldots, K\right\}$, meaning that there are $K_{1}$ noncognitive transmitters who are observed by $K-K_{1}$ cognitive transmitters (see Section V).

The paper is organized in the following form: In Section II, we introduce the system model and state important assumptions. In Section III, we review the non-cooperative games. In Section IV, we introduce the hierarchical game model and we characterize the hierarchical equilibrium. In Section V, we consider the two-level power control game model. Finally, we close with some concluding remarks in Section VII.

\section{System Model}

We consider the uplink of a single-cell network, in which $K$ users are simultaneously sending information to one base station (BS) in a decentralized manner. We assume that the users transmit their data over block Rayleigh flat-fading channels 
and the receiver knows on each block all the channel gains whereas each transmitter has only access to the knowledge of its own channel. The latter assumption is realistic in systems where the uplink-downlink channel reciprocity is valid and a reliable feedback mechanism is available or if the BS sends training sequences to the terminals. The signal received by the BS can be expressed as

$$
y=\sum_{k=1}^{K} h_{k} x_{k}+z
$$

where $x_{k}$ and $h_{k}$ are the input signal and channel coefficient for user $k$. We denote by $g_{k}=\left|h_{k}\right|^{2}$ the fading channel gain of user $k$, which varies over time but is assumed to be constant over each transmission block. The quantity $z$ represents a zero-mean additive white Gaussian noise (AWGN) with variance $\sigma^{2}$. The input signal $x_{k}$ can be further written as $x_{k}=\sqrt{p_{k}} s_{k}$ where $p_{k}$ and $s_{k}$ are the transmit power and data of user $k$. We will assume that the receiver applies single user decoding throughout this paper. This assumption is very relevant in practical wireless systems with limitations on the decoder complexity. Therefore, for any user $k \in\{1, \ldots, K\}$, the received single-user signal-to-noise plus interference ratio (SINR) can be expressed as

$$
\gamma_{k}=\frac{g_{k} p_{k}}{\sum_{j \neq k} g_{j} p_{j}+\sigma^{2}} .
$$

\section{REVIEW OF THE STANDARD NON-COOPERATIVE POWER CONTROL GAME}

In [2] the power control problem is modeled as strategicform non-cooperative game, which is given by: the set of players $\mathcal{K}=\{1, \ldots, K\}$ who are the transmitters here, the set of actions for each transmitter $\mathcal{P}_{k}=\left[0, P_{k}^{\max }\right]$, where $P_{k}^{\max }$ represents the transmit power constraint of player $k$, and the utility functions $u_{k}$. In this game, the objective of a transmitter is to choose his best power control policy (the best power level for each block) to maximize his utility function which is his energy-efficiency and is given by

$$
u_{k}\left(p_{1}, \ldots, p_{K}\right)=\frac{R_{k} f\left(\gamma_{k}\right)}{p_{k}}
$$

where $f(\cdot)$ is called efficiency function representing the packet success rate (which is assumed to be identical for all users in this paper), and $R_{k}$ is the transmission rate of user $k$. An important solution concept of the game under consideration is the Nash equilibrium (NE) [6], which is a fundamental concept in non-cooperative strategic games. It is a vector of strategies (or actions in our case) $\mathbf{p}^{\mathrm{NE}}=\left\{p_{1}^{\mathrm{NE}}, \ldots, p_{K}^{\mathrm{NE}}\right\}$, one for each player, such that no player has incentive to unilaterally change his strategy, i.e., $u_{k}\left(p_{k}^{\mathrm{NE}}, \mathbf{p}_{-k}^{\mathrm{NE}}\right) \geq u_{k}\left(p_{k}, \mathbf{p}_{-k}^{\mathrm{NE}}\right), \forall p_{k} \in \mathcal{P}_{k}$, where the $-k$ subscript on vector $\mathbf{p}$ stands for "except user $k$ ", i.e., $\mathbf{p}_{-k}=\left\{p_{1}, \ldots, p_{k-1}, p_{k+1}, \ldots, p_{K}\right\}$. From above, it is clear that an NE simply represents a particular "steady" state for a system which is stable to unilateral deviations. In many cases, an NE represents the result of learning and evolution of all involved players. It is therefore important to predict and characterize such points from the perspective of system design.

It is shown in [2] that, under certain conditions (given below), the NE of the game with utility (2) exists, and is given by

$$
p_{k}^{\mathrm{NE}}=\frac{\sigma^{2}}{g_{k}} \frac{\gamma^{*}}{1-(K-1) \gamma^{*}}, \forall k \in\{1, \ldots, K\}
$$

where $\gamma^{*}$ represents the SINR of user $k$ (which is the same for all users) and is the positive solution of the first order equation $x f^{\prime}(x)-f(x)=0$. This type of equation has a unique solution if the efficiency function $f(\cdot)$ is sigmoidal [7], and we will use this assumption throughout our paper. More precisely, the existence of a (pure) NE is insured if the condition $\gamma^{*}<\frac{1}{K-1}$ is satisfied. Note that when $\gamma^{*} \geq \frac{1}{K-1}$, this game still has an NE if the transmit power is limited, i.e., $p_{k} \in\left[0, P_{k}^{\max }\right]$. Therefore, if the users have not enough power to reach the SINR $\gamma^{*}$, they will all transmit with full power, which is also an equilibrium. For the sake of clarity, in this paper we will only consider the most interesting regime where the transmit powers are lower than their maximal levels. In this regime, even if a user has an infinite transmit power he will not necessarily use all of it. This is what (3) shows: each player tunes its transmit power such that its SINR is equal to a certain value $\gamma^{*}$. In this paper, we will denote by $\gamma^{\mathrm{NE}}$ and $u_{k}^{\mathrm{NE}}$ the SINR and utility that user $k$ obtains at the NE, respectively.

\section{Multi-LeVel Power CONTROL Game With ONE PLAYER PER LEVEL}

In this section, we introduce the hierarchy concept in the described non-cooperative game. We consider different hierarchical levels among the users, i.e., some users' decision priority is higher/lower than the others. It is easy to see that this hierarchy concept can be directly applied to the cognitive radio networks, where primary users can act in the most efficient way, taking into account the behaviors and impacts of secondary users. Specifically, we assume that the wireless system has $K$ users and $K$ hierarchical levels, i.e., one user per level. We also assume that the super leader is assigned with level index 1, and the last follower is assigned with level index $K$. Without loss of generality, we let the user index and level index be identical, i.e., user $k$ is assigned to level $k$, for any $k \in\{1, \ldots, K\}$.

\section{A. Characterization of hierarchical equilibria}

The equilibrium of this game is called hierarchical equilibrium (HE). In the following context, we will denote by $p_{k}^{\mathrm{HE}}, \gamma_{k}^{\mathrm{HE}}$ and $u_{k}^{\mathrm{HE}}$ the transmit power, SINR and utility of user $k$ at the HE, respectively. To characterize HE, we shall first look at the last follower (user $K$ ) who "makes the first move" to decide his transmit power $p_{K}^{\mathrm{HE}}$, according to the following maximization problem:

$$
\max _{p_{K}} \frac{f\left(\frac{g_{K} p_{K}}{\sum_{j \neq K} g_{j} p_{j}+\sigma^{2}}\right)}{p_{K}}
$$


whose solution $p_{K}^{\mathrm{HE}}$ satisfies

$$
\frac{g_{K} p_{K}}{\sum_{j \neq K} g_{j} p_{j}+\sigma^{2}}=\gamma_{K}^{\mathrm{HE}}
$$

where $\gamma_{K}^{\mathrm{HE}}$ is the solution of equation $x f^{\prime}(x)-f(x)=0$. Equation (5) implies that: at the HE, the last follower's (user $K$ ) best response is to tune his SINR to $\gamma_{K}^{\mathrm{HE}}$, which is equal to the SINR at the NE, i.e., $\gamma_{K}^{\mathrm{HE}}=\gamma^{\mathrm{NE}}$.

For the sake of simplicity of our presentation, we introduce $z_{k}$ and $\omega_{k}$ to simplify the notations:

$$
\begin{aligned}
z_{k} & \triangleq g_{k} p_{k}^{\mathrm{HE}} \\
\omega_{k} & \triangleq \sum_{j<k} g_{j} p_{j}^{\mathrm{HE}}+\sigma^{2}
\end{aligned}
$$

Then, (5) can be simply expressed as

$$
z_{K}=\gamma_{K}^{\mathrm{HE}} \omega_{K}
$$

and the similar expression holds for the second last follower (user $K-1$ ):

$$
z_{K-1}=\left[\frac{\gamma_{K-1}\left(1+\gamma_{K}\right)}{1-\gamma_{K-1} \gamma_{K}}\right] \omega_{K-1}
$$

In fact, this recursion can be proceeded until the super leader (user 1), and it can be further verified that such a relation always holds for some parameter $d_{k}$ (which will be defined later on):

$$
z_{k}=d_{k} \omega_{k}
$$

It turns out that this reasoning can be generalized by mathematical induction, which is the purpose of the following proposition.

Proposition 1. At the hierarchical equilibrium, the power allocation $\mathbf{p}^{H E}$ satisfies the following condition, for any $k$ and any $i$ with $k \geq i$

$$
p_{k}^{H E}= \begin{cases}d_{k} \omega_{i} / g_{k} & i=k \\ d_{k} \prod_{j=i}^{k-1}\left(1+d_{j}\right) \omega_{i} / g_{k} & i<k\end{cases}
$$

where

$$
d_{k}=\frac{\gamma_{k}^{H E}\left(1+b_{k}\right)}{1-\gamma_{k}^{H E} b_{k}}>0
$$

where

$$
b_{k}=\sum_{j>k}\left[d_{j} \prod_{k<n<j}\left(1+d_{n}\right)\right], \forall k<K
$$

with $b_{K}=0$, and $\gamma_{k}^{H E}$ is the solution of

$$
x\left(1-b_{k} x\right) f^{\prime}(x)-f(x)=0
$$

Proof: First, from (6), (7) and (9), we observe that

$$
\omega_{k}=\omega_{k-1}+z_{k-1}=\left(d_{k-1}+1\right) \omega_{k-1}
$$

which implies

$$
\omega_{k}=\prod_{j=i}^{k-1}\left(d_{j}+1\right) \omega_{i}, \forall i<k
$$

and from (9), we have

$$
z_{k}=d_{k} \prod_{j=i}^{k-1}\left(d_{j}+1\right) \omega_{i}, \forall i<k
$$

This shows that the relation (10) holds for some $d_{k}$.

Then, we show that (11) is the exact expression for $d_{k}$. When $k=K$, (8) gives $d_{k}=\gamma_{K}^{\mathrm{HE}}$; When $k<K$, we have

$$
z_{k}=\gamma_{k}^{\mathrm{HE}}\left(\sum_{j \neq k} z_{j}+\sigma^{2}\right)=\gamma_{k}^{\mathrm{HE}}\left(\omega_{k}+\sum_{j>k} z_{j}\right)
$$

By using (10) to (14), we obtain

$$
z_{k}=\gamma_{k}^{\mathrm{HE}}\left(\omega_{k}+b_{k} \omega_{k+1}\right)=\gamma_{k}^{\mathrm{HE}}\left[\omega_{k}+b_{k}\left(\omega_{k}+z_{k}\right)\right]
$$

where

$$
b_{k}=\sum_{j>k}\left[d_{j} \prod_{k<n<j}\left(d_{n}+1\right)\right]
$$

From (15), we have

$$
z_{k}=\frac{\gamma_{k}^{\mathrm{HE}}\left(1+b_{k}\right)}{1-\gamma_{k}^{\mathrm{HE}} b_{k}} \omega_{k}
$$

This confirms (11).

Finally, we prove that user $k$ 's SINR $\gamma_{k}^{\mathrm{HE}}$ must satisfy (13). From (15), we observe that the power-efficiency maximization problem of any user $k<K$ is given by

$$
\max _{p_{k}} \frac{f\left(\frac{g_{k} p_{k}}{\left(1+b_{k}\right) \omega_{k}+b_{k} g_{k} p_{k}}\right)}{p_{k}}
$$

Let $x=\frac{g_{k} p_{k}}{\left(1+b_{k}\right) \omega_{k}+b_{k} g_{k} p_{k}}$, then (16) is equivalent to

$$
\max _{x} \frac{\left(1-b_{k} x\right) f(x)}{\left(1+b_{k}\right) \omega_{k} x}
$$

The first order condition of (17) implies that $\gamma_{k}^{\mathrm{HE}}$ should satisfy

$$
x\left(1-b_{k} x\right) f^{\prime}(x)-f(x)=0
$$

This completes the proof.

From (12), we can observe that $b_{k}=\left(1+d_{k+1}\right) b_{k+1}+d_{k+1}$. This implies that $b_{i} \geq b_{j}$ for any $i$ and $j$ with $i<j$. Then from a simple geometrical argument, if $b_{i} \geq b_{j}$ holds, the solution $\gamma_{i}^{\mathrm{HE}}$ of the equation $x\left(1-b_{i} x\right) f^{\prime}(x)-f(x)=0$ is not higher than the solution $\gamma_{j}^{\mathrm{HE}}$ of $x\left(1-b_{j} x\right) f^{\prime}(x)-f(x)=0$, i.e., $\gamma_{i}^{\mathrm{HE}} \leq \gamma_{j}^{\mathrm{HE}}$. This means that, at the hierarchical equilibrium, a follower has better (or the same) SINR than a leader. Then, from equation (5), we can conclude that the SINR values of the $K$ users at the hierarchical equilibrium are organized from the lower level to the higher level of hierarchy, i.e.,

$$
\gamma_{1}^{\mathrm{HE}} \leq \gamma_{2}^{\mathrm{HE}} \leq \ldots \leq \gamma_{K}^{\mathrm{HE}}=\gamma^{\mathrm{NE}} .
$$

Proposition 2. At the hierarchical equilibrium, any user can obtain a better energy-efficient utility (2) by being in a lower hierarchical level. 
Proof: We consider a user $k$ and compare his utility if he is in the hierarchical level $i$ or in the level $j$ with $i<j$. We have $g_{k}=g_{i}=g_{j}$ and we compare the two utilities:

$$
\begin{aligned}
\frac{u_{i}^{\mathrm{HE}}}{u_{j}^{\mathrm{HE}}} & =\frac{f\left(\gamma_{i}\right) / p_{i}}{f\left(\gamma_{j}\right) / p_{j}}=\frac{f\left(\gamma_{i}\right) / g_{k} p_{i}}{f\left(\gamma_{j}\right) / g_{k} p_{j}}=\frac{f\left(\gamma_{i}\right) / g_{i} p_{i}}{f\left(\gamma_{j}\right) / g_{j} p_{j}} \\
& =\frac{f\left(\gamma_{i}\right)}{f\left(\gamma_{j}\right)} \frac{\left(1+\gamma_{i}\right) / \gamma_{i}}{\left(1+\gamma_{j}\right) / \gamma_{j}}=\frac{h\left(\gamma_{i}\right)}{h\left(\gamma_{j}\right)} \frac{\left(1+\gamma_{i}\right)}{\left(1+\gamma_{j}\right)},
\end{aligned}
$$

where $h(x) \triangleq f(x) / x$, which is a strictly increasing function on the interval $\left[0, \gamma^{\mathrm{NE}}\right]$ where $\gamma^{\mathrm{NE}}=\arg \max _{x} h(x)$ [7]. Then combining with $\gamma_{i}^{\mathrm{HE}} \leq \gamma_{j}^{\mathrm{HE}}$, we have that $\frac{u_{i}^{\mathrm{HE}}}{u_{j}^{\mathrm{HE}}} \leq 1$. This proves the result.

Considering the multi-level hierarchical game, we know that if the first player (the super leader) plays the Nash equilibrium, all other players will react by playing the Nash equilibrium. Then, the utility perceived by the super leader at the hierarchical equilibrium is almost equal to the one obtained at the Nash equilibrium of the system. Moreover, we have shown than the utility perceived is better for players at low levels than the super leader, their utility will be also better at the hierarchical equilibrium than at the Nash equilibrium.

Proposition 3. (Comparison to Nash equilibrium) At the hierarchical equilibrium, all users' transmit powers are reduced. Moreover, the hierarchical equilibrium Pareto-dominates the Nash equilibrium, if $\gamma_{1}^{\mathrm{HE}} \geq \gamma^{\star}=\arg \max _{x} f(x) / x$ holds.

Proof: We have

$$
\begin{aligned}
\sum_{k=1}^{K} \frac{1}{1+\gamma_{k}} & =\frac{\sum_{k=1}^{K}\left(\sigma^{2}+\sum_{j \neq k} g_{k} p_{k}\right)}{\sigma^{2}+\sum_{k=1}^{K} g_{k} p_{k}} \\
& =K-1+\frac{\sigma^{2}}{\sigma^{2}+\sum_{k=1}^{K} g_{k} p_{k}}
\end{aligned}
$$

From (18) and (19), we have

$$
\sum_{k=1}^{K} g_{k} p_{k}^{\mathrm{NE}} \geq \sum_{k=1}^{K} g_{k} p_{k}^{\mathrm{HE}}
$$

Suppose that $p_{k}^{\mathrm{NE}}<p_{k}^{\mathrm{HE}}$ for some user $k$. Then from (20), we have

$$
\sum_{j \neq k} g_{k} p_{k}^{\mathrm{NE}} \geq \sum_{j \neq k} g_{k} p_{k}^{\mathrm{HE}} \Longrightarrow \gamma^{\mathrm{NE}}<\gamma_{k}^{\mathrm{HE}}
$$

which is contradict to (18). This implies that the assumption of $p_{k}^{\mathrm{NE}}<p_{k}^{\mathrm{HE}}$ is not true. Therefore, we have $p_{k}^{\mathrm{NE}} \geq p_{k}^{\mathrm{HE}}$ for all $k \in\{1, \ldots, K\}$, i.e., the power level of any user $k$ at the HE is not greater than that at the NE. This proves our first claim. The following proof is not given in this paper due to lack of space.

\section{TWO-LEVEL POWER CONTROL GAME With $K_{1}$ LEADERS AND $K-K_{1}$ FOLLOWERS}

We consider the second scenario with $K_{1}$ non-cognitive transmitters who are observed by $K-K_{1}$ cognitive transmitters. In this context, the non-cognitive transmitters can be considered as leaders of a hierarchical non-cooperative game where the cognitive transmitters are the followers. This type of game leads to the concept of Stackelberg equilibrium [8]. Moreover, within each group, non-cognitive and cognitive, there exists a non-cooperative game that leads to the concept of Nash equilibrium. We denote by $\mathcal{L}$ (resp. $\mathcal{F}$ ) the set of mobiles that are in the group of leaders (resp. followers). This means that the size of $\mathcal{L}$ (resp. $\mathcal{F}$ ) is $K_{1}$ (resp. $K-K_{1}$ ).

We have the first result that shows the existence and uniqueness of a stable decision between all the transmitters. This stable decision is a Stackelberg equilibrium of the hierarchical non-cooperative game.

Proposition 4. (Existence and uniqueness of Stackelberg equilibrium) There exists a unique Stackelberg equilibrium $\mathbf{p}^{S E}$ in the proposed hierarchical game where:

$$
\begin{aligned}
p_{i}^{\mathrm{SE}} & =\frac{\sigma^{2}}{\left|h_{i}\right|^{2}} \frac{\gamma_{\mathcal{F}}^{\star}\left(1+\gamma_{\mathcal{L}}^{\star}\right)}{1-(K-1) \gamma_{\mathcal{F}}^{\star} \gamma_{\mathcal{L}}^{\star}-\left(K-K_{1}-1\right) \gamma_{\mathcal{L}}^{\star}}, \forall i \in \mathcal{L} \\
p_{j}^{\mathrm{SE}} & =\frac{\sigma^{2}}{\left|h_{j}\right|^{2}} \frac{\gamma_{\mathcal{L}}^{\star}\left(1+\gamma_{\mathcal{F}}^{\star}\right)}{1-(K-1) \gamma_{\mathcal{F}}^{\star} \gamma_{\mathcal{L}}^{\star}-\left(K-K_{1}-1\right) \gamma_{\mathcal{L}}^{\star}}, \forall j \in \mathcal{F}
\end{aligned}
$$

if the following (sufficient) conditions hold:

$$
\left\{\begin{aligned}
(1): & \frac{f^{\prime \prime}(0)}{f^{\prime}(0)} \geq 2 \frac{\left(K-K_{1}\right) \gamma_{\mathcal{L}}^{\star}}{1-\left(K-K_{1}-1\right) \gamma_{\mathcal{L}}^{\star}}, \text { and } \\
(2): & \phi(x)=x\left[1-\frac{\left(K-K_{1}\right) \gamma_{\mathcal{L}}^{\star}}{1-\left(K-K_{1}-1\right) \gamma_{\mathcal{L}}^{\star}} x\right] f^{\prime}(x)-f(x) \\
& \text { has a single maximum point in }\left(0, \gamma_{\mathcal{L}}^{\star}\right)
\end{aligned}\right.
$$

where $\gamma_{\mathcal{L}}^{\star}$ is the positive solution of $x f^{\prime}(x)-f(x)=0$ and $\gamma_{\mathcal{F}}^{\star}$ is the positive solution of $\phi(x)=0$.

Proof: Using the utility function defined by equation (2), we obtain from equation of the NE (3) that for all vector $p_{i}$, the optimal decision of a follower $j \neq i$, given the power of the leaders, is to choose the power

$$
p_{j}^{\mathrm{SE}}\left(p_{i}\right)=\frac{\gamma_{\mathcal{L}}^{\star}}{1-\left(K-K_{1}-1\right) \gamma_{\mathcal{L}}^{\star}} \frac{\left(\sigma^{2}+\sum_{i \in \mathcal{L}} g_{i} p_{i}\right)}{g_{j}} .
$$

This equation is given by a non-cooperative game between followers where the power of the leaders are included in the noise. Note that the $K-K_{1}-1$ term comes from interferences generated by all other followers than user $j$. The SINR of one leader $i \in \mathcal{L}$ is expressed by:

$$
\operatorname{SINR}_{i}=\frac{g_{i} p_{i}}{\sigma^{2}+\left(K-K_{1}\right) g_{j} p_{j}^{\mathrm{SE}}\left(p_{i}\right)+\sum_{k \in \mathcal{L} /\{i\}} g_{k} p_{k}} .
$$

We denote $\theta=\frac{\left(K-K_{1}\right) \gamma_{\mathcal{L}}^{*}}{1-\left(K-K_{1}-1\right) \gamma_{\mathcal{L}}^{*}}$ and then the SINR of a leader transmitter $i$ is

$$
\operatorname{SINR}_{i}=\frac{g_{i} p_{i}}{\sigma^{2}(1+\theta)+\theta g_{i} p_{i}+(1+\theta) \sum_{k \in \mathcal{L} /\{i\}} g_{k} p_{k}} .
$$

Plugging this value in the utility function of one leader, says user $i$, we obtain:

$$
\begin{aligned}
u_{i}\left(p_{i}\right) & =\frac{R_{i} f\left[\frac{g_{i} p_{i}}{\sigma^{2}(1+\theta)+\theta g_{i} p_{i}+(1+\theta) \sum_{k \in \mathcal{L} /\{i\}} g_{k} p_{k}}\right]}{p_{i}}, \\
& \triangleq \frac{R_{i} f\left[g\left(p_{i}, \mathbf{p}_{-i}\right)\right]}{p_{i}} .
\end{aligned}
$$


With respect to the action of the other leaders $\mathbf{p}_{-i}$, we have that the power used by the leader $i p_{i}^{\mathrm{SE}}$ has to verify $p_{i}^{\mathrm{SE}} \frac{\partial g\left(p_{i}^{\mathrm{SE}}, \mathbf{p}_{-i}\right)}{\partial p_{i}} f^{\prime}\left[g\left(p_{i}^{\mathrm{SE}}, \mathbf{p}_{-i}\right)\right]=f\left[g\left(p_{i}^{\mathrm{SE}}, \mathbf{p}_{-i}\right)\right]$. This equation is equivalent to finding $p_{i}$ such that

$g\left(p_{i}, \mathbf{p}_{-i}\right)\left(1-\theta g\left(p_{i}, \mathbf{p}_{-i}\right)\right) f^{\prime}\left[g\left(p_{i}, \mathbf{p}_{-i}\right)\right]=f\left[g\left(p_{i}, \mathbf{p}_{-i}\right)\right]$, because $p_{i}^{\mathrm{SE}} \frac{\partial g\left(p_{i}^{\mathrm{SE}}, \mathbf{p}_{-i}\right)}{\partial p_{i}}=g\left(p_{i}^{\mathrm{SE}}, \mathbf{p}_{-i}\right)\left(1-\theta g\left(p_{i}^{\mathrm{SE}}, \mathbf{p}_{-i}\right)\right)$. We define $x \triangleq \frac{g_{i} p_{i}}{\sigma^{2}(1+\theta)+\theta g_{i} p_{i}+(1+\theta) \sum_{k \in \mathcal{L} /\{i\}} g_{k} p_{k}}=g\left(p_{i}, \mathbf{p}_{-i}\right)$, then studying the existence and uniqueness issues for $p_{i}$ is equivalent to analyzing those of $x_{0}$ such that $\phi\left(x_{0}\right)=0$ with $\phi(x)=x(1-\theta x) f^{\prime}(x)-f(x)$ where:

1) $f$ is continuous over $[0,+\infty)$;

2) $f(0)=0$;

3) $\forall x \geq 0, f^{\prime}(x) \geq 0$;

4) $f$ is S-shaped, and there exists an $x^{\star}$ such that $\forall x \leq$ $x^{\star}, f^{\prime \prime}(x) \geq 0$ and $\forall x \geq x^{\star}, f^{\prime \prime}(x) \leq 0 ;$

5) $\lim _{x \rightarrow+\infty} f(x)=$ const;

6) $\lim _{p_{i} \rightarrow 0} u_{i}\left(p_{i}\right)=0$.

Recall that const $=1$ in [5]. This property is essential to insure the existence and uniqueness of $\gamma_{\mathcal{L}}^{\star}$. Therefore our problem reduces to find the sign of $\phi^{\prime}(x)$ for $x \geq 0$.

Existence of $x_{0}$ : We know $\phi(0)=0$ and $\phi(x)<$ $0, \forall x \geq \theta$. Therefore, if we can prove that $f$ is locally strictly positive on the interval $(0, \theta)$, the existence of $x_{0}$ will be guaranteed. A sufficient condition for the existence of $x_{0}$ is $\frac{f^{\prime \prime}(0)}{f^{\prime}(0)} \geq 2 \theta$. To check this, use $\phi^{\prime \prime}(x)=$ $-2 \theta f^{\prime}(x)+f^{\prime \prime}(x)+x\left[-4 \theta f^{\prime \prime}(x)+(1-\theta x) f^{\prime \prime \prime}(x)\right]$ and call for the Taylor-Lagrange theorem, i.e., there exists a constant $c \in(0, x)$ such that $\phi(x)=\phi^{\prime \prime}(0) \frac{x^{2}}{2}+\phi^{\prime \prime \prime}(c) \frac{c^{3}}{6}$. The quantity $\frac{c^{3}}{x^{2}} \leq x$ can be made arbitrary small in the neighborhood of zero. The proposed sufficient condition insures the convexity of $\phi$ and $\phi$ is therefore locally strictly positive.

Uniqueness of $x_{0}$ : It follows from the existence and the fact that $\phi$ is assumed to have a single stationary point in $\left(0, \gamma_{\mathcal{L}}^{\star}\right)$.

Each leader's transmit power at the equilibrium must satisfy $g_{i} p_{i}^{\mathrm{SE}}=g_{k} p_{k}^{\mathrm{SE}}$, for any leader index $i$ and $k$ with $i \neq k$. Then, the SINR of leader $i$ at equilibrium is

$$
\frac{g_{i} p_{i}^{\mathrm{SE}}}{\sigma^{2}(1+\theta)+\theta g_{i} p_{i}^{\mathrm{SE}}+(1+\theta)(n-1) g_{i} p_{i}^{\mathrm{SE}}}=\gamma_{\mathcal{F}}^{*} .
$$

Inverting this function we obtain:

$$
p_{i}^{\mathrm{SE}}=\frac{\sigma^{2}}{g_{i}} \frac{\gamma_{\mathcal{F}}^{\star}\left(1+\gamma_{\mathcal{L}}^{\star}\right)}{1-(K-1) \gamma_{\mathcal{F}}^{\star} \gamma_{\mathcal{L}}^{\star}-\left(K-K_{1}-1\right) \gamma_{\mathcal{L}}^{\star}} .
$$

Finally, taking equation (21), we obtain the transmit power of a follower $j \neq i$ :

$$
p_{j}^{S E}=\frac{\sigma^{2}}{g_{j}} \frac{\gamma_{\mathcal{L}}^{\star}\left(1+\gamma_{\mathcal{F}}^{\star}\right)}{1-(K-1) \gamma_{\mathcal{F}}^{\star} \gamma_{\mathcal{L}}^{\star}-\left(K-K_{1}-1\right) \gamma_{\mathcal{L}}^{\star}} .
$$

This completes the proof.

The powers at the Stackelberg equilibrium depends on the repartition between leaders and followers in the system, that is the number of leaders $K_{1}$. Then, in the numerical illustrations section, we present global performance of the system as the social welfare depending on the number of cognitive transmitters.

\section{NUMERICAL ILLUSTRATIONS}

We illustrate the second scenario with the impact of the number of cognitive transmitters on the global metric of the system which is the social welfare, i.e. the summation of the utilities of all players. We consider the efficiency function $f(x)=\left(1-e^{-x}\right)^{100}, K=10$ transmitters totally symmetric, i.e. all transmitters have the same channel conditions and rates.

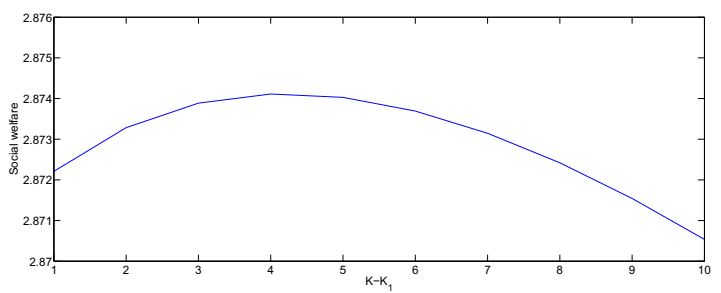

Fig. 1. Social welfare depending on the number of cognitive transmitters.

We observe on figure 1 that the social welfare of the system is maximized when there are $K-K_{1}=4$ cognitive transmitters and $K_{1}=6$ non-cognitive ones. This simple result hints that the choice of the number of cognitive transmitters has an impact on the global network performance, and apparently there exists an optimum choice for this number.

\section{CONCLUSION}

This paper studies the hierarchical equilibria in the context of cognitive networks where multiple cognitive and noncognitive transmitters coexist and competitively maximize their individual energy efficiencies. In the case of multiple hierarchy levels, an interesting result showed that followers (non-cognitive users) can obtain better energy efficiency than leaders (cognitive users). In the case of two hierarchy levels, it was shown that there exists an optimal partition between all users in the networks. These results provide some insights to the system design perspective of future cognitive networks.

\section{ACKNOWLEDGEMENT}

This work is support by l'Agence Nationale de la Recherche (ANR) on the reference ANR-09-VERS-001: ECOSCELLS.

\section{REFERENCES}

[1] S. Lasaulce, M. Debbah, and E. Altman, "Methodologies for analyzing equilibria in wireless games", IEEE Signal Processing Magazine special issue on Game Theory, vol. 26, no. 5, pp. 51-52, Sep. 2009.

[2] D. J. Goodman and N. B. Mandayam, "Power Control for Wireless Data", IEEE Personal Commun., vol. 7, no. 2, pp. 48-54, April 2000.

[3] C. U. Saraydar, N. B. Mandayam and D. J. Goodman, "Efficient Power Control via Pricing in Wireless Data Networks", IEEE Trans. on Commun., vol. 50, no. 2, pp. 291-303, Feb. 2002.

[4] S. Lasaulce, Y. Hayel, R. El Azouzi and M. Debbah, "Introducing Hierachy in Energy Games", IEEE Trans. on Wireless Commun., vol. 8, pp. 3833-3843, July 2009.

[5] F. Meshkati, M. Chiang, H. V. Poor, and S. C. Schwartz, "A Gametheoretic Approach to Energy-efficient Power Control in Multi-carrier CDMA Systems", IEEE J. Select. Areas Commun., vol. 24, no. 6, pp. 1115-1129, June 2006

[6] J. F. Nash, "Equilibrium Points in N-points Games", Proc. of the National Academy of Science, vol. 36, no. 1, pp. 48-49, Jan. 1950.

[7] V. Rodriguez, "An Analytical Foundation for Resource Management in Wireless Communication", IEEE Proc. of Globecom, 2003.

[8] H. Von Stackelberg, "The Theory of the Market Economy", Oxford University, Press, Oxford, England, 1952. 